ANNALES

POLONICI MATHEMATICI

$81.3(2003)$

\title{
Existence theory for single and multiple solutions to singular positone boundary value problems for the delay one-dimensional $p$-Laplacian
}

\author{
by Daqing Jiang (Changchun), XiaOJie Xu (Changchun),
} Donal O'Regan (Galway) and Ravi P. Agarwal (Melbourne, FL)

\begin{abstract}
The existence of single and multiple nonnegative solutions for singular positone boundary value problems to the delay one-dimensional $p$-Laplacian is discussed. Throughout our nonlinearity $f(\cdot, y)$ may be singular at $y=0$.
\end{abstract}

1. Introduction. Recently in the literature on the theory of functional differential equations many authors have discussed singular and nonsingular positone boundary value problems (i.e. problems where $f$ takes nonnegative values) for second order functional differential equations of the form

$$
x^{\prime \prime}(t)+f\left(t, x_{t}\right)=0,
$$

or

$$
x^{\prime \prime}(t)+f(t, x(\tau(t)))=0 ;
$$

for example see $[1,8,11,12,18]$ and the references therein. In $[8,12,18]$, the problem discussed does not allow singularities. In $[1,7,11]$, the existence of one nonnegative solution of singular positone problems for second order functional differential equations is studied. As pointed out by the authors of [7], these problems are motivated by applications in physics, applied mathematics and variational problems in control theory.

In this paper, we study the existence of single and multiple nonnegative solutions to the singular positone boundary value problem

2000 Mathematics Subject Classification: Primary 34B15.

Key words and phrases: existence, multiple nonnegative solutions, singular boundary value problem, delay differential equation.

The work was supported by NNSF of China. 


$$
\left\{\begin{array}{l}
\left(\Phi\left(y^{\prime}\right)\right)^{\prime}+q(t) f(t, y(t-\tau))=0, \quad t \in(0,1) \backslash\{\tau\} \\
y(t)=\xi(t), \quad-\tau \leq t \leq 0 \\
y(1)=0
\end{array}\right.
$$

where $\Phi(s)=|s|^{p-2} s, p>1,0<\tau<1, \xi \in C[-\tau, 0], \xi(t)>0$ on $[-\tau, 0)$ and $\xi(0)=0$. Here our nonlinearity $f(t, y)$ may be singular at $y=0$.

In $[2,3](p=2, \tau=0)$, R. P. Agarwal and D. O'Regan showed that the singular positone problem has single and two nonnegative solutions by using a Leray-Schauder alternative and a general fixed point theorem in cones. In $[4,5,9](p=2, \tau=0)$, the authors used Krasnosel'skil's fixed point theorem in a cone to establish the existence of two nonnegative solutions to singular positone boundary value problems. In $[10,13,16](\tau=0)$, the problem discussed can only have singularities at $t=0$ or $t=1$. However, no paper to date has discussed the existence of single and multiple nonnegative solutions to singular positone boundary value problems for the delay one-dimensional $p$-Laplacian. This paper attempts to fill this gap in the literature. Existence will be established by using a Leray-Schauder alternative [15] and Krasnosel'skiì's fixed point theorem in a cone [6].

We next state a result from $[2,3]$ which will be needed in Section 3.

LEMMA 1.1. Let

$$
K_{[0,1]}=\{y \in C[0,1]: y \geq 0 \text { and concave on }[0,1]\} \text {. }
$$

If $y \in K_{[0,1]}$, then

$$
y(t) \geq t(1-t)\|y\|_{[0,1]} \quad \text { for } t \in[0,1],
$$

where $\|y\|_{[0,1]}=\sup _{t \in[0,1]}|y(t)|$.

Finally, for completeness we state Krasnosel'skiı̌'s fixed point theorem in a cone [6].

LEMMA 1.2. Let $E=(E,\|\cdot\|)$ be a Banach space and let $K \subset E$ be a cone in $E$. Assume $\Omega_{1}, \Omega_{2}$ are open subsets of $E$ with $0 \in \Omega_{1}, \bar{\Omega}_{1} \subset \Omega_{2}$, and let

$$
A: K \cap\left(\bar{\Omega}_{2} \backslash \Omega_{1}\right) \rightarrow K
$$

be a completely continuous operator such that either

(i) $\|A y\| \leq\|y\| \forall y \in K \cap \partial \Omega_{1}$ and $\|A y\| \geq\|y\| \forall y \in K \cap \partial \Omega_{2}$, or

(ii) $\|A y\| \geq\|y\| \forall y \in K \cap \partial \Omega_{1}$ and $\|A y\| \leq\|y\| \forall y \in K \cap \partial \Omega_{2}$.

Then $A$ has a fixed point in $K \cap\left(\bar{\Omega}_{2} \backslash \Omega_{1}\right)$.

2. Existence principles. In this section, we establish existence principles for the boundary value problem 


$$
\left\{\begin{array}{l}
\left(\Phi\left(y^{\prime}\right)\right)^{\prime}+F(t, y(t-\tau))=0, \quad 0<t<1 \\
y(t)=A(t), \quad-\tau \leq t \leq 0 \\
y(1)=B
\end{array}\right.
$$

where $\Phi(s)=|s|^{p-2} s, p>1,0<\tau<1, A \in C[-\tau, 0]$ and $A(0)=A$. Suppose the following two conditions are satisfied:

(A1) $\quad F:(0,1) \times \mathbb{R} \rightarrow \mathbb{R}$ is continuous;

(A2) for each $r>0$ there exists $h_{r} \in C((0,1),[0, \infty)) \cap L^{1}[0,1]$ such that $|u| \leq r$ implies $|F(t, u)| \leq h_{r}(t)$ for $t \in(0,1)$.

Suppose that $D \subset E:=C[-\tau, 1]$ is a bounded set, and there exists a constant $r>0$ such that $\|u\| \leq r$ for $u \in \bar{D}$, where

$$
\|u\|=\max _{t \in[-\tau, 1]}|u(t)| .
$$

Thus $|F(t, u(t-\tau))| \leq h_{r}(t)$ for $u \in \bar{D}$.

For each fixed $y \in D$, we consider the boundary value problem

$$
\left\{\begin{array}{l}
\left(\Phi\left(w^{\prime}\right)\right)^{\prime}+F(t, y(t-\tau))=0, \quad 0<t<1 \\
w(t)=A(t), \quad-\tau \leq t \leq 0 \\
w(1)=B
\end{array}\right.
$$

Then (2.2) is equivalent to

$$
w(t)=(T y)(t):= \begin{cases}A+\int_{0}^{t} \Phi^{-1}\left(C_{y}+\int_{x}^{1} F(s, y(s-\tau)) d s\right) d x, \\ A(t), & 0 \leq t \leq 1, \\ & -\tau \leq t \leq 0,\end{cases}
$$

where $C_{y}=\Phi\left(w^{\prime}(1)\right)$ is a solution of the equation

$$
Z\left(C_{y}\right):=\int_{0}^{1} \Phi^{-1}\left(C_{y}+\int_{x}^{1} F(s, y(s-\tau)) d s\right) d x=B-A .
$$

Lemma 2.1. For each $y \in D,(2.4)$ has a unique solution $C_{y} \in \mathbb{R}$.

Proof. Fix $y \in D$. Then, by the definition of $Z\left(C_{y}\right)$,

$$
\int_{0}^{1} \Phi^{-1}\left(C_{y}-\int_{x}^{1} h_{r}(s) d s\right) d x \leq Z\left(C_{y}\right) \leq \int_{0}^{1} \Phi^{-1}\left(C_{y}+\int_{x}^{1} h_{r}(s) d s\right) d x
$$

for all $C_{y} \in \mathbb{R}$. Because $\Phi^{-1}$ is a continuous, strictly increasing function on $\mathbb{R}$ with $\Phi^{-1}(\mathbb{R})=\mathbb{R}$, so is $Z$ (for each fixed $y \in D$ ). Thus, there exists a unique $C_{y} \in \mathbb{R}$ satisfying (2.4).

From Lemma 2.1, we conclude that $T: D \rightarrow E$ is well defined.

Lemma 2.2. $T: \bar{D} \rightarrow E$ is continuous and compact. 
Proof. Fix $y \in \bar{D}$ and let $C_{y} \in \mathbb{R}$ be the unique solution of (2.4) corresponding to $y$. From (2.4), by the first mean value theorem, there exists a $\xi \in[0,1]$ such that

$$
\Phi^{-1}\left(C_{y}+\int_{\xi}^{1} F(s, y(s-\tau)) d s\right)=B-A,
$$

i.e.,

$$
C_{y}=\Phi(B-A)-\int_{\xi}^{1} F(s, y(s-\tau)) d s
$$

Then

$$
\left|C_{y}\right| \leq \Phi(|B-A|)+\int_{0}^{1} h_{r}(s) d s=: M(r),
$$

and

$$
\left|C_{y}+\int_{t}^{1} F(s, y(s-\tau)) d s\right| \leq M(r) \quad \text { for } t \in[0,1] .
$$

From (2.3), (2.6) and (2.7), we have

$$
|w(t)| \leq|A|+M^{1 /(p-1)}(r), \quad\left|w^{\prime}(t)\right| \leq M^{1 /(p-1)}(r), \quad 0 \leq t \leq 1,
$$

where $M(r)$ is a positive constant independent of $y \in \bar{D}$ and $t \in[0,1]$.

Since $w(t)=A(t)$ for $t \in[-\tau, 0]$, the Arzelà-Ascoli Theorem guarantees that $T: \bar{D} \rightarrow E$ is compact.

Now assume that $y_{0}, y_{n} \in \bar{D}$ and $y_{n} \rightarrow y_{0}$ in $\bar{D}$. Then

$$
(2.8)^{n} \quad\left(T y_{n}\right)(t)= \begin{cases}A+\int_{0}^{t} \Phi^{-1}\left(C_{y_{n}}+\int_{x}^{1} F\left(s, y_{n}(s-\tau)\right) d s\right) d x \\ A(t), & 0 \leq t \leq 1, \\ -\tau \leq t \leq 0\end{cases}
$$

where $C_{y_{n}}(n=\{0,1, \ldots\})$ is the unique solution of the equation

$$
Z\left(C_{y_{n}}\right):=\int_{0}^{1} \Phi^{-1}\left(C_{y_{n}}+\int_{x}^{1} F\left(s, y_{n}(s-\tau)\right) d s\right) d x=B-A .
$$

From (2.6), we know that $\left|C_{y_{n}}\right| \leq M(r), n=0,1,2, \ldots$, where $M(r)$ is independent of $y_{n}$. Suppose that $C^{*} \in[-M(r), M(r)]$ is an accumulation point of $\left\{C_{y_{n}}\right\}$. Then there is a subsequence of $\left\{C_{y_{n}}\right\},\left\{C_{y_{n(j)}}\right\}$, which converges to $C^{*}$. It follows from $(2.9)^{n(j)}$ that

$$
\int_{0}^{1} \Phi^{-1}\left(C^{*}+\int_{x}^{1} F\left(s, y_{0}(s-\tau)\right) d s\right) d x=B-A .
$$


This shows that $C^{*}=C_{y_{0}}$, by Lemma 2.1. Hence $\left\{C_{y_{n}}\right\}$ has a unique accumulation point, and hence $C_{y_{n}} \rightarrow C_{y_{0}}$. Thus, from $(2.8)^{n}$ and $(2.9)^{n}$, we have

$$
\lim _{n \rightarrow \infty}\left(T y_{n}\right)(t)=\left(T y_{0}\right)(t), \quad t \in[-\tau, 1]
$$

This shows that $T: \bar{D} \rightarrow E$ is continuous. The proof of the lemma is now complete.

Since $D$ is an arbitrary bounded subset in $C[0,1]$, we have

Lemma 2.3. $T: E \rightarrow E$ is completely continuous.

We obtain the following general existence principles for (2.1) by using the Schauder fixed point theorem and a nonlinear alternative of Leray-Schauder type.

TheOrem 2.1. Suppose (A1) and (A2) hold. In addition suppose there is a constant $M>\sup _{t \in[-\tau, 0]}|A(t)|+|B|$, independent of $\lambda$, with

$$
\|y\|=\max _{t \in[-\tau, 1]}|y(t)| \neq M
$$

for any solution $y \in C[-\tau, 1] \cap C^{1}[0,1]$ to

$$
\left\{\begin{array}{l}
\left(\Phi\left(y^{\prime}\right)\right)^{\prime}+\lambda^{p-1} F(t, y(t-\tau))=0, \quad 0<t<1 \\
y(t)=\lambda A(t), \quad-\tau \leq t \leq 0 \\
y(1)=\lambda B
\end{array}\right.
$$

for each $\lambda \in(0,1)$. Then (2.1) has a solution y with $\|y\| \leq M$.

Proof. Notice that $(2.11)_{\lambda}$ is equivalent to the fixed point problem

$$
y=\lambda T y, \quad u \in C[-\tau, 1],
$$

where $T$ is as in (2.3). Set $U=\{u \in C[-\tau, 1]:\|u\|<M\}$. Since $T$ : $C[-\tau, 1] \rightarrow C[-\tau, 1]$ is completely continuous, the nonlinear alternative [15] guarantees that $T$ has a fixed point, i.e., $(2.11)_{1}$ has a solution in $\bar{U}$.

TheOREm 2.2. Suppose (A1) and (A2) hold. In addition suppose there is a constant $M>\sup _{t \in[-\tau, 0]}|A(t)|+|B|$, independent of $\lambda$, with

$$
\|y\|=\max _{t \in[-\tau, 1]}|y(t)| \neq M
$$

for any solution $y \in C[-\tau, 1] \cap C^{1}[0,1]$ to

$$
\left\{\begin{array}{l}
\left(\Phi\left(y^{\prime}-(1-\lambda)(B-A)\right)\right)^{\prime}+\lambda^{p-1} F(t, y(t-\tau))=0, \quad 0<t<1, \\
y(t)=A(t), \quad-\tau \leq t \leq 0, \\
y(1)=B,
\end{array}\right.
$$

for each $\lambda \in(0,1)$. Then (2.1) has a solution $y$ with $\|y\| \leq M$.

Proof. Notice that $(2.13)_{\lambda}$ is equivalent to the fixed point problem

$$
y=(1-\lambda) Q+\lambda T y,
$$


where

$$
Q(t)= \begin{cases}A(1-t)+B t, & 0 \leq t \leq 1 \\ A(t), & -\tau \leq t \leq 1\end{cases}
$$

Set $U=\{u \in C[-\tau, 1]:\|u\|<M\}$. Since $T: C[-\tau, 1] \rightarrow C[-\tau, 1]$ is completely continuous, the nonlinear alternative guarantees that $T$ has a fixed point, i.e., $(2.14)_{1}$ has a solution in $\bar{U}$.

TheOREM 2.3. Suppose that (A1) holds, and there exists $h \in C((0,1)$, $[0, \infty)) \cap L^{1}[0,1]$ with $|F(t, u)| \leq h(t)$ for $t \in(0,1)$ and $u \in \mathbb{R}$. Then $(2.1)$ has a solution $y$.

Proof. Solving (2.1) is equivalent to the fixed point problem $u=T u$. Since $T: C[-\tau, 1] \rightarrow C[-\tau, 1]$ is compact, the result follows from Schauder's fixed point theorem.

REMARK 2.1. Theorems 2.1-2.3 extend results in $[2](p=2, \tau=0)$ and [14] $(\tau=0)$.

3. Delay differential equations. In this section we will discuss (1.1). We shall assume the following conditions:

$$
\begin{aligned}
& \xi \in C[-\tau, 0], \xi(t)>0 \text { on }[-\tau, 0) \text { and } \xi(0)=0, \\
& q \in C(0,1) \cap L^{1}[0,1] \text { with } q>0 \text { on }(0,1), \\
& f:[0,1] \times(0, \infty) \rightarrow(0, \infty) \text { is continuous, }
\end{aligned}
$$

$f(t, u) \leq g(u)+h(u)$ on $[0,1] \times(0, \infty)$ with $g>0$ continuous and nonincreasing on $(0, \infty), h \geq 0$, continuous on $[0, \infty)$ and $h / g$ nondecreasing on $(0, \infty)$

$$
\begin{aligned}
& \text { there exists } K_{0} \text { with } g(a b) \leq K_{0} g(a) g(b) \text { for all } a>0, b>0, \\
& \int_{0}^{\tau} q(s) f(s, \xi(s-\tau)) d s<\infty
\end{aligned}
$$$$
\int_{0}^{\tau} q(s) g(\xi(s-\tau)) d s<\infty, \int_{\tau}^{1} q(s) g((s-\tau)(1+\tau-s)) d s<\infty .
$$

TheOREm 3.1. Suppose (3.1)-(3.7) hold. In addition suppose the following conditions are satisfied:

(3.8) for each constant $H>0$ there exists a function $\psi_{H}$ continuous on $[0,1]$ and positive on $(0,1)$ such that $f(t, u) \geq \psi_{H}(t)$ on $[0,1] \times$ $(0, H]$

(3.9) there exists $r>b_{0}=\int_{0}^{\tau} \Phi^{-1}\left(\int_{s}^{\tau} q(x) f(x, \xi(x-\tau)) d x\right) d s$ with $r / \Phi^{-1}(g(r)+h(r))>a_{0}$, where $\Phi^{-1}(u)=|u|^{1 /(p-1)} \operatorname{sgn} u$ is the inverse to $\Phi(u)$, and

$$
a_{0}=K_{0}^{1 /(p-1)} \int_{\tau}^{1} \Phi^{-1}\left(\int_{\tau}^{s} q(x) g((x-\tau)(1+\tau-x)) d x\right) d s .
$$


Then (1.1) has a solution $y \in C[-\tau, 1] \cap C^{1}[0,1]$ with $y(t)>0$ for $t \in(0,1)$ and $\|y-w\|<r$, where

$$
w(t)= \begin{cases}0, & 0 \leq t \leq 1 \\ \xi(t), & -\tau \leq t \leq 0\end{cases}
$$

Proof. Choose $\delta \in(0, r)$ with

$$
\begin{aligned}
& \frac{r-\delta}{\Phi^{-1}(g(r)+h(r))}>a_{0}, \\
& \delta+\int_{0}^{\tau} \Phi^{-1}\left(\int_{s}^{\tau} q(x) f(x, \xi(x-\tau)) d x\right) d s<r .
\end{aligned}
$$

Let $m_{0} \in\{1,2, \ldots\}$ be chosen so that $1 / m_{0}<\delta$ and

$$
\delta+\int_{0}^{\tau} \Phi^{-1}\left(\int_{s}^{\tau} q(x) f(x, 1 / m+\xi(x-\tau)) d x\right) d s<r \quad \text { for } m \in N_{0},
$$

where $N_{0}=\left\{m_{0}, m_{0}+1, \ldots\right\}$. To show (1.1) has a nonnegative solution we will look at the boundary value problem

$$
\left\{\begin{array}{l}
\left(\Phi\left(y^{\prime}\right)\right)^{\prime}+q(t) f(t, y(t-\tau)+w(t-\tau))=0, \quad t \in(0,1) \backslash\{\tau\} \\
y(t)=0, \quad-\tau \leq t \leq 0 \\
y(1)=0
\end{array}\right.
$$

We will show, using Theorem 2.2, that there exists a solution $y$ to (3.11) with $y(t)>0$ for $t \in(0,1)$ and $y(t)=0$ for $t \in[-\tau, 0]$ and $\|y\|<r$. If this is true then $u(t)=y(t)+w(t),-\tau \leq t \leq 1$, is a nonnegative solution (positive on $(0,1) \cup[-\tau, 0))$ of $(1.1)$ and $\|u-w\|<r$. Therefore, we focus on (3.11).

The idea is to show first that

$$
\left\{\begin{array}{l}
\left(\Phi\left(y^{\prime}\right)\right)^{\prime}+q(t) F(t, y(t-\tau)+w(t-\tau))=0, \quad t \in(0,1) \backslash\{\tau\} \\
y(t)=1 / m, \quad-\tau \leq t \leq 0 \\
y(1)=1 / m,
\end{array}\right.
$$

has a solution $y_{m}$ for each $m \in N_{0}$ with $y_{m}(t) \geq 1 / m$ for $t \in[0,1]$ and $\left\|y_{m}\right\|<r$, where

$$
F(t, u)= \begin{cases}f(t, u), & u \geq 1 / m \\ f(t, 1 / m), & u \leq 1 / m\end{cases}
$$

We will apply Theorem 2.2. Consider the family of problems

$(3.13)_{\lambda}^{m}\left\{\begin{array}{l}\left(\Phi\left(y^{\prime}\right)\right)^{\prime}+\lambda^{p-1} q(t) F(t, y(t-\tau)+w(t-\tau))=0, \quad t \in(0,1) \backslash\{\tau\}, \\ y(t)=1 / m, \quad-\tau \leq t \leq 0, \\ y(1)=1 / m,\end{array}\right.$

for $0<\lambda<1$. Let $y \in C[-\tau, 1] \cap C^{1}[0,1]$ be any solution of $(3.13)_{\lambda}^{m}$. Then $\left(\Phi\left(y^{\prime}\right)\right)^{\prime} \leq 0$ on $(0,1)$ and it is easy to check that $y$ is concave on $[0,1]$. 
Thus, $y(t) \geq 1 / m$ on $[0,1]$. Also there exists $\sigma \in(0,1)$ with $y^{\prime}(t) \geq 0$ on $(0, \sigma), y^{\prime}(t) \leq 0$ on $(\sigma, 1)$ and $y(\sigma)=\|y\|$. Moreover, by Lemma 1.1,

$$
y(t) \geq t(1-t) y(\sigma)=t(1-t)\|y\|, \quad t \in[0,1] .
$$

Therefore, $(3.13)_{\lambda}^{m}$ is equivalent to

$$
(3.15)_{\lambda}^{m} \quad y(t)=\left\{\begin{array}{rr}
1 / m+\lambda \int_{0}^{t} \Phi^{-1}\left(\int_{s}^{\sigma} q(x) F(x, y(x-\tau)+w(x-\tau)) d x\right) d s \\
1 / m+\lambda \int_{t}^{1} \Phi^{-1}\left(\int_{\sigma}^{s} q(x) F(x, y(x-\tau)+w(x-\tau)) d x\right) d s \\
1 / m, & \sigma \leq t \leq 1 \\
& -\tau \leq t \leq 0
\end{array}\right.
$$

where $\sigma$ satisfies

$$
\begin{aligned}
\int_{0}^{\sigma} \Phi^{-1}\left(\int_{s}^{\sigma} q(x) F(x, y\right. & (x-\tau)+w(x-\tau)) d x) d s \\
= & \int_{\sigma}^{1} \Phi^{-1}\left(\int_{\sigma}^{s} q(x) F(x, y(x-\tau)+w(x-\tau)) d x\right) d s .
\end{aligned}
$$

We next claim that

$$
\|y\|=\max _{t \in[-\tau, 1]}|y(t)| \neq r
$$

for any solution $y$ to $(3.15)_{\lambda}^{m}$. Suppose this is false, i.e. suppose there exists $\lambda$ and a solution $y$ to $(3.15)_{\lambda}^{m}$ such that $\|y\|=r$. Then by (3.14), $y(t) \geq t(1-t) r$ for $t \in[0,1]$, and so $y(x-\tau) \geq(x-\tau)(1+\tau-x) r$ for $x \in[\tau, 1]$. Notice also that

$$
\begin{array}{ll}
F(x, y(x-\tau)+w(x-\tau))=f(x, 1 / m+\xi(x-\tau)) & \text { for } x \in(0, \tau), \\
F(x, y(x-\tau)+w(x-\tau))=f(x, y(x-\tau)) & \text { for } x \in(\tau, 1) .
\end{array}
$$

Let $\sigma$ be as in $(3.15)_{\lambda}^{m}$. If $0<\sigma \leq \tau$, then

$$
\begin{aligned}
r=y(\sigma) & =1 / m+\lambda \int_{0}^{\sigma} \Phi^{-1}\left(\int_{s}^{\sigma} q(x) F(x, y(x-\tau)+w(x-\tau)) d x\right) d s \\
& =1 / m+\lambda \int_{0}^{\sigma} \Phi^{-1}\left(\int_{s}^{\sigma} q(x) f(x, y(x-\tau)+w(x-\tau)) d x\right) d s \\
& =1 / m+\lambda \int_{0}^{\sigma} \Phi^{-1}\left(\int_{s}^{\sigma} q(x) f(x, 1 / m+\xi(x-\tau)) d x\right) d s \\
& \leq \delta+\int_{0}^{\tau} \Phi^{-1}\left(\int_{s}^{\tau} q(x) f(x, 1 / m+\xi(x-\tau)) d x\right) d s .
\end{aligned}
$$

This contradicts (3.10b), so (3.16) is satisfied. 
If $\tau \leq \sigma<1$, then

$$
\begin{aligned}
r=y(\sigma)= & 1 / m+\lambda \int_{\sigma}^{1} \Phi^{-1}\left(\int_{\sigma}^{s} q(x) F(x, y(x-\tau)+w(x-\tau)) d x\right) d s \\
= & 1 / m+\lambda \int_{\sigma}^{1} \Phi^{-1}\left(\int_{\sigma}^{s} q(x) f(x, y(x-\tau)) d x\right) d s \\
\leq & \delta+\int_{\tau}^{1} \Phi^{-1}\left(\int_{\tau}^{s} q(x)[g(y(x-\tau))+h(y(x-\tau))] d x\right) d s \\
= & \delta+\int_{\tau}^{1} \Phi^{-1}\left(\int_{\tau}^{s} q(x) g(y(x-\tau))\left(1+\frac{h(y(x-\tau))}{g(y(x-\tau))}\right) d x\right) d s \\
\leq & \delta+\Phi^{-1}\left(1+\frac{h(r)}{g(r)}\right) \int_{\tau}^{1} \Phi^{-1}\left(\int_{\tau}^{s} q(x) g((x-\tau)(1+\tau-x) r) d x\right) d s \\
\leq & \delta+\Phi^{-1}\left(K_{0}\right) \Phi^{-1}(g(r)+h(r)) \\
& \times \int_{\tau}^{1} \Phi^{-1}\left(\int_{\tau}^{s} q(x) g((x-\tau)(1+\tau-x)) d x\right) d s \\
= & \delta+a_{0} \Phi^{-1}(g(r)+h(r)) .
\end{aligned}
$$

This contradicts (3.10a), so (3.16) is satisfied.

Now Theorem 2.2 implies that $(3.12)^{m}$ has a solution $y_{m}$ with $\left\|y_{m}\right\| \leq r$. In fact (as above)

$$
1 / m \leq y_{m}(t)<r \quad \text { for } t \in[0,1]
$$

Next we obtain a sharper lower bound on $y_{m}$, namely we show that there exists a constant $k>0$, independent of $m$, with

$$
y_{m}(t) \geq k t(1-t) \quad \text { for } t \in[0,1] \text {. }
$$

To see this notice that (3.8) guarantees the existence of a function $\psi_{M}$ continuous on $[0,1]$ and positive on $(0,1)$ with $F\left(t, y_{m}(t-\tau)+w(t-\tau)\right)=$ $f\left(t, y_{m}(t-\tau)+w(t-\tau)\right) \geq \psi_{M}(t)$ for $t \in[0,1]$, where $M:=r+\sup _{t \in[-\tau, 0]} \xi(t)$ $\geq\left\|y_{m}\right\|+\|w\|$. Now, let $V_{M} \in C[0,1] \cap C^{1}[0,1]$ be the unique solution to the problem

$$
\left\{\begin{array}{l}
\left(\Phi\left(y^{\prime}\right)\right)^{\prime}+q(t) \psi_{M}(t)=0, \quad 0<t<1 \\
y(0)=0, \quad y(1)=0
\end{array}\right.
$$

Since $\left(\Phi\left(V_{M}^{\prime}\right)\right)^{\prime}<0$ on $(0,1), V_{M}$ is concave on $[0,1]$ and $\left\|V_{M}\right\|_{[0,1]}=$ $\sup _{t \in[0,1]}\left|V_{M}(t)\right|>0$. Moreover, by Lemma 1.1,

$$
V_{M}(t) \geq t(1-t)\left\|V_{M}\right\|_{[0,1]}, \quad t \in[0,1] .
$$


Since $F\left(t, y_{m}(t-\tau)+w(t-\tau)\right) \geq \psi_{M}(t)$ for $t \in[0,1]$, we claim that

$$
y_{m}(t) \geq V_{M}(t), \quad t \in[0,1] .
$$

Suppose (3.20) is false, i.e. assume $y_{m}(t)<V_{M}(t)$ for some $t \in(0,1)$. Since $y_{m}(0)>V_{M}(0)$ and $y_{m}(1)>V_{M}(1)$, the function $u(t)=V_{M}(t)-y_{m}(t)$ would have a positive maximum at a point $t_{0} \in(0,1)$. Hence $V_{M}^{\prime}\left(t_{0}\right)=y_{m}^{\prime}\left(t_{0}\right)$, and there exist $0 \leq t_{1}<t_{0}<t_{2} \leq 1$ such that $u\left(t_{1}\right)=u\left(t_{2}\right)=0$ and $u(t)>0$ on $\left(t_{1}, t_{2}\right)$. Notice that

$$
\begin{aligned}
& {\left[\Phi\left(V_{M}^{\prime}(t)\right)\right]^{\prime}-\left[\Phi\left(y_{m}^{\prime}(t)\right)\right]^{\prime}} \\
& \quad=-q(t) \psi_{M}(t)+q(t) F\left(t, y_{m}(t-\tau)+w(t-\tau)\right) \geq 0, \quad \forall t \in(0,1) .
\end{aligned}
$$

Integrate both sides of the above inequality with respect to $t$ from $t_{0}$ to $t \in\left(t_{0}, t_{2}\right)$, to get

$$
\left[\Phi\left(V_{M}^{\prime}(t)\right)\right]-\left[\Phi\left(V_{M}^{\prime}\left(t_{0}\right)\right)\right] \geq\left[\Phi\left(y_{m}^{\prime}(t)\right)\right]-\left[\Phi\left(y_{m}^{\prime}\left(t_{0}\right)\right)\right] \quad \text { for all } t \in\left(t_{0}, t_{2}\right) .
$$

That is,

$$
u^{\prime}(t)=V_{M}^{\prime}(t)-y_{m}^{\prime}(t) \geq 0 \quad \text { for } t \in\left(t_{0}, t_{2}\right),
$$

and so $u\left(t_{0}\right) \leq u\left(t_{2}\right)=0$, a contradiction.

Now (3.20) together with (3.19) implies (3.17) with $k=\left\|V_{M}\right\|_{[0,1]}$.

We shall now obtain a solution to (3.11) by means of the Arzelà-Ascoli Theorem, as a limit of solutions of $(3.12)^{\mathrm{m}}$. We will show that

$$
\left\{y_{m}\right\}_{m \in N_{0}} \text { is a bounded, equicontinuous family on }[-\tau, 1] \text {. }
$$

Of course $\left\|y_{m}\right\|<r$ implies $\left\{y_{m}\right\}_{m \in N_{0}}$ is uniformly bounded. To show equicontinuity notice that

$$
y_{m}(t)=\left\{\begin{array}{rr}
1 / m+\int_{0}^{t} \Phi^{-1}\left(\int_{s}^{\sigma_{m}} q(x) f\left(x, y_{m}(x-\tau)+w(x-\tau)\right) d x\right) d s, & 0 \leq t \leq \sigma_{m}, \\
1 / m+\int_{t}^{1} \Phi^{-1}\left(\int_{\sigma_{m}}^{s} q(x) f\left(x, y_{m}(x-\tau)+w(x-\tau)\right) d x\right) d s, & \sigma_{m} \leq t \leq 1, \\
1 / m, & -\tau \leq t \leq 0,
\end{array}\right.
$$

where $\sigma_{m}$ satisfies

$$
\begin{aligned}
& \int_{0}^{\sigma_{m}} \Phi^{-1}\left(\int_{s}^{\sigma_{m}} q(x) f\left(x, y_{m}(x-\tau)+w(x-\tau)\right) d x\right) d s \\
& =\int_{\sigma_{m}}^{1} \Phi^{-1}\left(\int_{\sigma_{m}}^{s} q(x) f\left(x, y_{m}(x-\tau)+w(x-\tau)\right) d x\right) d s
\end{aligned}
$$


and

$$
y_{m}^{\prime}(t)=\left\{\begin{array}{r}
\Phi^{-1}\left(\int_{t}^{\sigma_{m}} q(x) f\left(x, y_{m}(x-\tau)+w(x-\tau)\right) d x\right) \\
-\Phi^{-1}\left(\int_{\sigma_{m}}^{t} q(x) f\left(x, y_{m}(x-\tau)+w(x-\tau)\right) d x\right) \\
\quad \sigma_{m} \leq t \leq 1,
\end{array}\right.
$$

where $\sigma_{m} \in(0,1)$ is such that $y_{m}\left(\sigma_{m}\right)=\left\|y_{m}\right\|$. So for $t \in[0,1]$ we have

$$
\begin{aligned}
\left|y_{m}^{\prime}(t)\right| \leq & \Phi^{-1}\left(\int_{0}^{1} q(x) f\left(x, y_{m}(x-\tau)+w(x-\tau)\right) d x\right) \\
= & \Phi^{-1}\left(\int_{0}^{\tau} q(x) f(x, 1 / m+\xi(x-\tau)) d x+\int_{\tau}^{1} q(x) f\left(x, y_{m}(x-\tau)\right) d x\right) \\
\leq & \Phi^{-1}\left(\int_{0}^{\tau} q(x) g(x, \xi(x-\tau))\left\{1+\frac{h(\delta+\|\xi\|)}{g(\delta+\|\xi\|)}\right\} d x\right. \\
& \left.+K_{0} g(k)\left\{1+\frac{h(r)}{g(r)}\right\} \int_{\tau}^{1} q(x) g((x-\tau)(1+\tau-x)) d x\right) .
\end{aligned}
$$

Since $y_{m}(t)=1 / m$ for $t \in[-\tau, 0]$, this implies that $\left\{y_{m}\right\}_{m \in N_{0}}$ is an equicontinuous family on $[-\tau, 1]$.

Now the Arzelà-Ascoli Theorem guarantees the existence of a subsequence $N^{+}$of $N_{0}$ and a function $y \in C[-\tau, 1]$ with $y_{m}$ converging to $y$ uniformly on $[-\tau, 1]$ as $m \rightarrow \infty$ through $N^{+}$. Moreover, there exists a constant $\sigma$ with $\sigma_{m}$ converging to $\sigma$ as $m \rightarrow \infty$ through $N^{+}$. Also $y(t)=0$ for $t \in[-\tau, 0], y(1)=0$ and $k t(1-t) \leq y(t)$.

Notice $y_{m}(t) \geq k t(1-t)$ for $0 \leq t \leq 1$, and $y_{m}(x-\tau) \geq k(x-\tau)(1+\tau-x)$ for $x \in[\tau, 1]$. Notice also that

$$
f\left(x, y_{m}(x-\tau)+w(x-\tau)\right)=f(x, 1 / m+\xi(x-\tau)) \quad \text { for } x \in(0, \tau),
$$

and

$$
\begin{aligned}
f\left(x, y_{m}(x-\tau)+w(x-\tau)\right) & =f\left(x, y_{m}(x-\tau)\right) \\
& \leq K_{0}\left\{1+\frac{h(\delta+r)}{g(\delta+r)}\right\} g(k) g((x-\tau)(1+\tau-x))
\end{aligned}
$$

for $x \in(\tau, 1)$. 
Now, let $m \rightarrow \infty$ (through $N^{+}$) in (3.22) to obtain (here we use the Lebesgue dominated convergence theorem)

$$
y(t)= \begin{cases}\int_{0}^{t} \Phi^{-1}\left(\int_{s}^{\sigma} q(x) f(x, y(x-\tau)+w(x-\tau)) d x\right) d s, & 0 \leq t \leq \sigma \\ \int_{t}^{1} \Phi^{-1}\left(\int_{\sigma}^{s} q(x) f(x, y(x-\tau)+w(x-\tau)) d x\right) d s, & \sigma \leq t \leq 1, \\ 0, & -\tau \leq t \leq 0\end{cases}
$$

where $\sigma$ satisfies

$$
\begin{aligned}
\int_{0}^{\sigma} \Phi^{-1}\left(\int_{s}^{\sigma} q(x) f(x, y\right. & (x-\tau)+w(x-\tau)) d x) d s \\
& =\int_{\sigma}^{1} \Phi^{-1}\left(\int_{\sigma}^{s} q(x) f(x, y(x-\tau)+w(x-\tau)) d x\right) d s .
\end{aligned}
$$

From (3.24) we deduce immediately that $y \in C[-\tau, 1] \cap C^{1}[0,1]$ is a nonnegative solution of $(3.11)$ with $y(t) \geq k t(1-t)$ on $[0,1]$ and $\|y\| \leq r$ (in fact $\|y\|<r$ as above).

REMARK 3.1. If we replace (3.9) with

$$
\sup _{c \in\left(b_{0}, \infty\right)} \frac{c}{\Phi^{-1}(g(c)+h(c))}>a_{0}
$$

(where $a_{0}, b_{0}$ are defined by (3.9)), the conclusion of Theorem 3.1 is still true.

Example 3.1. Consider the boundary value problem $(p=2)$

$$
\left\{\begin{array}{l}
y^{\prime \prime}(t)+\sigma\left(y^{-\alpha}(t-\tau)+y^{\beta}(t-\tau)\right)=0, \quad t \in(0,1) \backslash\{\tau\} \\
y(t)=-t, \quad-\tau \leq t \leq 0 \\
y(1)=0, \quad 0<\alpha<1, \beta \geq 0,0<\tau<1
\end{array}\right.
$$

where $\sigma>0$ is such that

$$
\sigma<\sup _{c \in(0, \infty)} \min \left(\frac{c}{b_{1}}, \frac{c^{\alpha+1}}{a_{1}\left(1+c^{\alpha+\beta}\right)}\right)
$$

with

$$
\begin{aligned}
b_{1} & =\int_{0}^{\tau} x\left[(\tau-x)^{-\alpha}+(\tau-x)^{\beta}\right] d x, \\
a_{1} & =\int_{\tau}^{1}(1-x)(x-\tau)^{-\alpha}(1+\tau-x)^{-\alpha} d x .
\end{aligned}
$$

Then (3.26) has a solution $y$ with $y(t)>0$ for $t \in(0,1)$. 
REMARK 3.2. If $\beta<1$ then (3.27) is automatically satisfied.

To see this we will apply Theorem 3.1 with

$$
g(y)=y^{-\alpha}, \quad h(y)=y^{\beta}, \quad q(t)=\sigma, \quad \xi(t)=-t, \quad K_{0}=1 .
$$

Clearly (3.1)-(3.8) hold. Next notice that

$$
\begin{aligned}
b_{0} & =\int_{0}^{\tau} \int_{s}^{\tau} q(x) f(x, \xi(x-\tau)) d x d s \\
& =\sigma \int_{0}^{\tau} x\left[(\tau-x)^{-\alpha}+(\tau-x)^{\beta}\right] d x=\sigma b_{1}
\end{aligned}
$$

and

$$
\begin{aligned}
a_{0} & =\sigma \int_{\tau}^{1} \int_{\tau}^{s}(x-\tau)^{-\alpha}(1+\tau-x)^{-\alpha} d x d s \\
& =\sigma \int_{\tau}^{1}(1-x)(x-\tau)^{-\alpha}(1+\tau-x)^{-\alpha} d x=\sigma a_{1},
\end{aligned}
$$

so (3.9) is true since (3.27) implies there exists $r>0$ such that

$$
\sigma<\min \left(\frac{r}{b_{1}}, \frac{r^{\alpha+1}}{a_{1}\left(1+r^{\alpha+\beta}\right)}\right),
$$

i.e.,

$$
r>b_{0} \text { and } \frac{r}{g(r)+h(r)}>a_{0} .
$$

Thus all the conditions of Theorem 3.1 are satisfied, so existence is guaranteed.

Next we establish the existence of multiple nonnegative solutions to (1.1) using Lemma 1.2.

Theorem 3.2. Suppose (3.1)-(3.7) and (3.9) hold. In addition suppose the following conditions are satisfied:

(3.28) there exists $0<a<(1-\tau) / 2$ (fixed hereafter), a continuous, nonincreasing function $g_{1}:(0, \infty) \rightarrow(0, \infty)$, and a continuous function $h_{1}:[0, \infty) \rightarrow(0, \infty)$ with $h_{1} / g_{1}$ nondecreasing on $(0, \infty)$ and $f(t, u) \geq g_{1}(u)+h_{1}(u)$ for $(t, u) \in[\tau+a, 1-a] \times(0, \infty)$,

(3.29) there exists $0<R_{1}<r<R_{2}$ with

$$
\frac{R_{i}}{\Phi^{-1}\left(g_{1}\left(R_{i}\right)\right) \Phi^{-1}\left(1+\frac{h_{1}\left(a(a+\tau) R_{i}\right)}{g_{1}\left(a(a+\tau) R_{i}\right)}\right)}<B_{0}
$$


for $i=1,2$, and

$$
B_{0}=\frac{1}{2} \min _{\tau+a \leq t \leq 1-a}\left\{\int_{\tau+a}^{t} \Phi^{-1}\left(\int_{s}^{t} q(x) d x\right) d s+\int_{t}^{1-a} \Phi^{-1}\left(\int_{t}^{s} q(x) d x\right) d s\right\} .
$$

Then (1.1) has two nonnegative solutions $y_{i} \in C[-\tau, 1] \cap C^{1}[0,1]$ with $y_{i}(t)$ $>0$ for $t \in(0,1)$ and $R_{1}<\left\|y_{1}-w\right\|<r<\left\|y_{2}-w\right\|<R_{2}, i=1,2$, where $w$ is as in Theorem 3.1.

Proof. To show (1.1) has two nonnegative solutions we will look at the boundary value problem

$$
\left\{\begin{array}{l}
\left(\Phi\left(y^{\prime}\right)\right)^{\prime}+q(t) f(t, y(t-\tau)+w(t-\tau))=0, \quad t \in(0,1) \backslash\{\tau\} \\
y(t)=0, \quad-\tau \leq t \leq 0 \\
y(1)=0
\end{array}\right.
$$

We will show, using Lemma 2.1, that there exist two solutions $y_{i}(i=1,2)$ to (3.30) with $y_{i}(t)>0$ for $t \in(0,1)$ and $y_{i}(t)=0$ for $t \in[-\tau, 0]$. If this is true then $u_{i}(t)=y_{i}(t)+w(t),-\tau \leq t \leq 1$, are nonnegative solutions (positive on $(0,1) \cup[-\tau, 0))$ of (1.1). Therefore, we focus on (3.30).

Let $E=(C[-\tau, 1],\|\cdot\|)$ with the norm $\|u\|:=\sup \{|u(t)|:-\tau \leq t \leq 1\}$, and let $K=\{u \in C[-\tau, 1]: u$ is a nonnegative concave function on $[0,1]$, $u(t)=0$ for $t \in[-\tau, 0], u(1)=0\}$. Clearly $K$ is a cone in $E$ and $\|u\|=$ $\|u\|_{[0,1]}$ for $u \in K$. Moreover, by Lemma 1.1, if $u \in K$, then

$$
u(t) \geq t(1-t)\|u\|, \quad t \in[0,1] .
$$

First we will show that there exists a solution $y_{2}$ to (3.30) with $y_{2}(t)>0$ for $t \in(0,1)$ and $r<\left\|y_{2}\right\|<R_{2}$. Let

$$
\Omega_{1}=\{u \in E:\|u\|<r\}, \quad \Omega_{2}=\left\{u \in E:\|u\|<R_{2}\right\} .
$$

Next let $A: K \cap\left(\bar{\Omega}_{2} \backslash \Omega_{1}\right) \rightarrow E$ be defined by

$$
(A y)(t)= \begin{cases}\int_{0}^{t} \Phi^{-1}\left(\int_{s}^{\sigma_{y}} q(x) f(x, y(x-\tau)+w(x-\tau)) d x\right) d s, & 0 \leq t \leq \sigma_{y}, \\ \int_{t}^{1} \Phi^{-1}\left(\int_{\sigma_{y}}^{s} q(x) f(x, y(x-\tau)+w(x-\tau)) d x\right) d s, \\ 0, & \sigma_{y} \leq t \leq 1, \\ 0 & -\tau \leq t \leq 0,\end{cases}
$$

where $\sigma_{y}$ is a solution of the equation

$$
z_{0}(\sigma):=\int_{0}^{\sigma} \Phi^{-1}\left(\int_{s}^{\sigma} q(x) f(x, y(x-\tau)+w(x-\tau)) d x\right) d s
$$




$$
=\int_{\sigma}^{1} \Phi^{-1}\left(\int_{\sigma}^{s} q(x) f(x, y(x-\tau)+w(x-\tau)) d x\right) d s=: z_{1}(\sigma), \quad 0 \leq \sigma \leq 1 .
$$

First we show $A$ is well defined. To see this notice that if $y \in K \cap\left(\bar{\Omega}_{2} \backslash \Omega_{1}\right)$ then $r \leq\|y\| \leq R_{2}$ and $y(t) \geq t(1-t)\|y\| \geq t(1-t) r$ for $0 \leq t \leq 1$, and so $y(x-\tau) \geq(x-\tau)(1+\tau-x) r$ for $x \in[\tau, 1]$. Also notice that

$$
f(x, y(x-\tau)+w(x-\tau))=f(x, \xi(x-\tau)) \quad \text { for } x \in(0, \tau),
$$

and

$$
\begin{aligned}
f(x, y( & x-\tau)+w(x-\tau))=f(x, y(x-\tau)) \\
& \leq g(y(x-\tau))+h(y(x-\tau)) \\
& =g(y(x-\tau))\left\{1+\frac{h(y(x-\tau))}{g(y(x-\tau))}\right\} \\
& \leq g((x-\tau)(1+\tau-x) r)\left\{1+\frac{h\left(R_{2}\right)}{g\left(R_{2}\right)}\right\} \\
& \leq K_{0} g((x-\tau)(1+\tau-x)) g(r)\left\{1+\frac{h\left(R_{2}\right)}{g\left(R_{2}\right)}\right\} \quad \text { for } x \in(\tau, 1) .
\end{aligned}
$$

These inequalities together with (3.6)-(3.7) guarantee that the operator $A: K \cap\left(\bar{\Omega}_{2} \backslash \Omega_{1}\right) \rightarrow E$ is well defined. Moreover $\sigma_{y} \in(0,1)$ is the unique solution of $(3.32)$ since $z_{0}(\sigma)$ is a strictly increasing continuous function defined on $[0,1]$ with $z_{0}(0)=0$, and $z_{1}(\sigma)$ is a strictly decreasing continuous function defined on $[0,1]$ with $z_{1}(1)=0$.

Next we show $A: K \cap\left(\bar{\Omega}_{2} \backslash \Omega_{1}\right) \rightarrow K$. If $y \in K \cap\left(\bar{\Omega}_{2} \backslash \Omega_{1}\right)$, then

(i) $(A y)^{\prime}(t)= \begin{cases}\Phi^{-1}\left(\int_{t}^{\sigma_{y}} q(x) f(x, y(x-\tau)+w(x-\tau)) d x\right), & 0 \leq t \leq \sigma_{y}, \\ -\Phi^{-1}\left(\int_{\sigma_{y}}^{t} q(x) f(x, y(x-\tau)+w(x-\tau)) d x\right), & \sigma_{y} \leq t \leq 1,\end{cases}$

(ii) $\quad\left\{\Phi\left[(A y)^{\prime}(t)\right]\right\}^{\prime}=-q(t) f(t, y(t-\tau)+w(t-\tau)), \quad t \in(0,1) \backslash\{\tau\}$,

(iii) $\quad\|A y\|=(A y)\left(\sigma_{y}\right), \quad(A y)(t)=0$ for $t \in[-\tau, 0], \quad(A y)(1)=0$.

This shows that $(A y)(t)$ is a nonnegative concave function defined on $[0,1]$, with $(A y)(t)=0$ for $t \in[-\tau, 0]$ and $(A y)(1)=0$. Consequently, $A y \in K$, so $A: K \cap\left(\bar{\Omega}_{2} \backslash \Omega_{1}\right) \rightarrow K$.

Next we show $A: K \cap\left(\bar{\Omega}_{2} \backslash \Omega_{1}\right) \rightarrow K$ is continuous and compact. Let $y_{n}, y_{0} \in K \cap\left(\bar{\Omega}_{2} \backslash \Omega_{1}\right)$ with $\left\|y_{n}-y_{0}\right\| \rightarrow 0$ as $n \rightarrow \infty$. Of course $r \leq\left\|y_{n}\right\|=\left\|y_{n}\right\|_{[0,1]} \leq R_{2}, r \leq\left\|y_{0}\right\|=\left\|y_{0}\right\|_{[0,1]} \leq R_{2}, y_{n}(t) \geq t(1-t) r$ for $0 \leq t \leq 1$, and so $y_{n}(x-\tau) \geq(x-\tau)(1+\tau-x) r$ for $x \in[\tau, 1]$. Notice also 
that

$$
\begin{aligned}
& f\left(x, y_{n}(x-\tau)+w(x-\tau)\right)=f(x, \xi(x-\tau)) \quad \text { for } x \in(0, \tau), \\
& f\left(x, y_{n}(x-\tau)+w(x-\tau)\right)=f\left(x, y_{n}(x-\tau)\right) \\
& \rightarrow f\left(x, y_{0}(x-\tau)\right) \quad \text { as } n \rightarrow \infty, x \in(\tau, 1),
\end{aligned}
$$

and

$$
f\left(x, y_{n}(x-\tau)+w(x-\tau)\right) \leq K_{0}\left\{1+\frac{h\left(R_{2}\right)}{g\left(R_{2}\right)}\right\} g(r) g((x-\tau)(1+\tau-x))
$$

for $x \in(\tau, 1)$. Suppose that $\sigma^{*} \in[0,1]$ is an accumulation point of $\left\{\sigma_{y_{n}}\right\}$, where $\sigma_{y_{n}} \in(0,1), n=1,2, \ldots$, satisfies the equation

$$
\begin{aligned}
\int_{0}^{\sigma_{y_{n}}} \Phi^{-1} & \left(\int_{s}^{\sigma_{y_{n}}} q(x) f\left(x, y_{n}(x-\tau)+w(x-\tau)\right) d x\right) d s \\
& =\int_{\sigma_{y_{n}}}^{1} \Phi^{-1}\left(\int_{\sigma_{y_{n}}}^{s} q(x) f\left(x, y_{n}(x-\tau)+w(x-\tau)\right) d x\right) d s .
\end{aligned}
$$

There exists a subsequence $\left\{\sigma_{y_{n_{k}}}\right\}$ which converges to $\sigma^{*}$. Insert $y_{n_{k}}$ and $\sigma_{y_{n_{k}}}$ into $(3.33)^{n_{k}}$ and then let $k \rightarrow \infty$ (using the Lebesgue dominated convergence theorem) to obtain

$$
\begin{aligned}
\int_{0}^{\sigma^{*}} \Phi^{-1}\left(\int_{s}^{\sigma^{*}} q(x) f\left(x, y_{0}(x-\tau)+w(x-\tau)\right) d x\right) d s \\
=\int_{\sigma^{*}}^{1} \Phi^{-1}\left(\int_{\sigma^{*}}^{s} q(x) f\left(x, y_{0}(x-\tau)+w(x-\tau)\right) d x\right) d s
\end{aligned}
$$

This shows that $\sigma^{*}=\sigma_{y_{0}}$ by the uniqueness of $\sigma_{y_{0}}$. Thus $\left\{\sigma_{y_{n}}\right\}$ has a unique accumulation point, and hence $\sigma_{y_{n}} \rightarrow \sigma_{y_{0}}$. Now the Lebesgue dominated convergence theorem guarantees that

$$
\begin{aligned}
& \lim _{n \rightarrow \infty}\left(A y_{n}\right)(t) \\
& =\lim _{n \rightarrow \infty} \begin{cases}\int_{0}^{t} \Phi^{-1}\left(\int_{s}^{\sigma_{y_{n}}} q(x) f\left(x, y_{n}(x-\tau)+w(x-\tau)\right) d x\right) d s, & 0 \leq t \leq \sigma_{y_{n}}, \\
\int_{t}^{1} \Phi^{-1}\left(\int_{\sigma_{y_{n}}}^{s} q(x) f\left(x, y_{n}(x-\tau)+w(x-\tau)\right) d x\right) d s, & \sigma_{y_{n}} \leq t \leq 1, \\
0, & -\tau \leq t \leq 0\end{cases} \\
& =\left(A y_{0}\right)(t), \quad t \in[0,1] .
\end{aligned}
$$

This shows that $A: K \cap\left(\bar{\Omega}_{2} \backslash \Omega_{1}\right) \rightarrow K$ is continuous. Also for $y \in K \cap$ $\left(\bar{\Omega}_{2} \backslash \Omega_{1}\right)$ we have 


$$
\begin{aligned}
\|A y\| & =(A y)\left(\sigma_{y}\right)=\int_{0}^{\sigma_{y}} \Phi^{-1}\left(\int_{s}^{\sigma_{y}} q(x) f(x, y(x-\tau)+w(x-\tau)) d x\right) d s \\
\leq & \Phi^{-1}\left(\int_{0}^{1} q(x) f(x, y(x-\tau)+w(x-\tau)) d x\right) d s \\
= & \Phi^{-1}\left(\int_{0}^{\tau} q(x) f(x, \xi(x-\tau)) d x+\int_{\tau}^{1} q(x) f(x, y(x-\tau)) d x\right) \\
\leq & \Phi^{-1}\left(\int_{0}^{\tau} q(x) f(x, \xi(x-\tau)) d x\right. \\
& \left.+K_{0} g(r)\left[1+\frac{h\left(R_{2}\right)}{g\left(R_{2}\right)}\right] \int_{\tau}^{1} q(x) g((x-\tau)(1+\tau-x)) d x\right),
\end{aligned}
$$

and for $t \in[0,1]$ we have

$$
\begin{aligned}
\left|(A y)^{\prime}(t)\right| \leq & \Phi^{-1}\left(\int_{0}^{1} q(x) f(x, y(x-\tau)+w(x-\tau)) d x\right) \\
\leq & \Phi^{-1}\left(\int_{0}^{\tau} q(x) f(x, \xi(x-\tau)) d x\right. \\
& \left.+K_{0} g(r)\left[1+\frac{h\left(R_{2}\right)}{g\left(R_{2}\right)}\right] \int_{\tau}^{1} q(x) g((x-\tau)(1+\tau-x)) d x\right) .
\end{aligned}
$$

Now the Arzelà-Ascoli Theorem guarantees that $A: K \cap\left(\bar{\Omega}_{2} \backslash \Omega_{1}\right) \rightarrow K$ is compact.

We now show

$$
\|A y\|<\|y\| \quad \text { for } y \in K \cap \partial \Omega_{1} .
$$

To see this, let $y \in K \cap \partial \Omega_{1}$. Then $\|y\|=\|y\|_{[0,1]}=r$ and $y(t) \geq t(1-t) r$ for $t \in[0,1]$, and so $y(x-\tau) \geq(x-\tau)(1+\tau-x) r$ for $x \in[\tau, 1]$.

If $0<\sigma_{y} \leq \tau$, then

$$
\begin{aligned}
\|A y\|=(A y)\left(\sigma_{y}\right) & =\int_{0}^{\sigma_{y}} \Phi^{-1}\left(\int_{s}^{\sigma_{y}} q(x) f(x, y(x-\tau)+w(x-\tau)) d x\right) d s \\
& =\int_{0}^{\sigma_{y}} \Phi^{-1}\left(\int_{s}^{\sigma_{y}} q(x) f(x, \xi(x-\tau)) d x\right) d s \\
& \leq \int_{0}^{\tau} \Phi^{-1}\left(\int_{s}^{\tau} q(x) f(x, \xi(x-\tau)) d x\right) d s<r=\|y\|,
\end{aligned}
$$

so (3.34) is satisfied. 
If $\tau \leq \sigma_{y}<1$, then

$$
\begin{aligned}
\|A y\| & =(A y)\left(\sigma_{y}\right)=\int_{\sigma_{y}}^{1} \Phi^{-1}\left(\int_{\sigma_{y}}^{s} q(x) f(x, y(x-\tau)+w(x-\tau)) d x\right) d s \\
& =\int_{\sigma_{y}}^{1} \Phi^{-1}\left(\int_{\sigma_{y}}^{s} q(x) f(x, y(x-\tau)) d x\right) d s \\
& \leq \int_{\tau}^{1} \Phi^{-1}\left(\int_{\tau}^{s} q(x)[g(y(x-\tau))+h(y(x-\tau))] d x\right) d s \\
& =\int_{\tau}^{1} \Phi^{-1}\left(\int_{\tau}^{s} q(x) g(y(x-\tau))\left(1+\frac{h(y(x-\tau))}{g(y(x-\tau))}\right) d x\right) d s \\
& \leq \Phi^{-1}\left(1+\frac{h(r)}{g(r)}\right) \int_{\tau}^{1} \Phi^{-1}\left(\int_{\tau}^{s} q(x) g((x-\tau)(1+\tau-x) r) d x\right) d s \\
& \leq \Phi^{-1}\left(K_{0}\right) \Phi^{-1}(g(r)+h(r)) \int_{\tau}^{1} \Phi^{-1}\left(\int_{\tau}^{s} q(x) g((x-\tau)(1+\tau-x)) d x\right) d s \\
& =a_{0} \Phi^{-1}(g(r)+h(r))<r,
\end{aligned}
$$

so (3.34) is again satisfied.

Next we show

$$
\|A y\|>\|y\| \quad \text { for } y \in K \cap \partial \Omega_{2} .
$$

To see this, let $y \in K \cap \partial \Omega_{2}$ so $\|y\|=\|y\|_{[0,1]}=R_{2}$ and $y(t) \geq t(1-t) R_{2}$ for $t \in[0,1]$, so that $y(x-\tau) \geq(x-\tau)(1+\tau-x) R_{2}$ for $x \in[\tau, 1]$. Moreover, $y(x-\tau) \geq a(a+\tau) R_{2}$ for $x \in[\tau+a, 1-a]$, since $a \in(0,(1-\tau) / 2)$.

If $0<\sigma_{y} \leq \tau+a$, then

$$
\begin{aligned}
\|A y\| & =(A y)\left(\sigma_{y}\right)=\int_{\sigma_{y}}^{1} \Phi^{-1}\left(\int_{\sigma_{y}}^{s} q(x) f(x, y(x-\tau)+w(x-\tau)) d x\right) d s \\
& \geq \int_{\tau+a}^{1-a} \Phi^{-1}\left(\int_{\tau+a}^{s} q(x) f(x, y(x-\tau)+w(x-\tau)) d x\right) d s \\
& =\int_{\tau+a}^{1-a} \Phi^{-1}\left(\int_{\tau+a}^{s} q(x) f(x, y(x-\tau)) d x\right) d s \\
& \geq \int_{\tau+a}^{1-a} \Phi^{-1}\left(\int_{\tau+a}^{s} q(x) g_{1}(y(x-\tau))\left(1+\frac{h_{1}(y(x-\tau))}{g_{1}(y(x-\tau))}\right) d x\right) d s
\end{aligned}
$$




$$
\begin{aligned}
& \geq \Phi^{-1}\left(g_{1}\left(R_{2}\right)\right) \Phi^{-1}\left(1+\frac{h_{1}\left(a(a+\tau) R_{2}\right)}{g_{1}\left(a(a+\tau) R_{2}\right)}\right) \int_{\tau+a}^{1-a} \Phi^{-1}\left(\int_{\tau+a}^{s} q(x) d x\right) d s \\
& \geq B_{0} \Phi^{-1}\left(g_{1}\left(R_{2}\right)\right) \Phi^{-1}\left(1+\frac{h_{1}\left(a(a+\tau) R_{2}\right)}{g_{1}\left(a(a+\tau) R_{2}\right)}\right) \\
& >R_{2}=\|y\| .
\end{aligned}
$$$$
\text { If } \tau+a \leq \sigma_{y} \leq 1-a \text {, then }
$$

$$
\begin{aligned}
& 2\|A y\|=\int_{0}^{\sigma_{y}} \Phi^{-1}\left(\int_{s}^{\sigma_{y}} q(x) f(x, y(x-\tau)+w(x-\tau)) d x\right) d s \\
& +\int_{\sigma_{y}}^{1} \Phi^{-1}\left(\int_{\sigma_{y}}^{s} q(x) f(x, y(x-\tau)+w(x-\tau)) d x\right) d s \\
& \geq \int_{\tau+a}^{\sigma_{y}} \Phi^{-1}\left(\int_{s}^{\sigma_{y}} q(x) f(x, y(x-\tau)) d x\right) d s \\
& +\int_{\sigma_{y}}^{1-a} \Phi^{-1}\left(\int_{\sigma_{y}}^{s} q(x) f(x, y(x-\tau)) d x\right) d s \\
& \geq \int_{\tau+a}^{\sigma_{y}} \Phi^{-1}\left(\int_{s}^{\sigma_{y}} q(x) g_{1}(y(x-\tau))\left(1+\frac{h_{1}(y(x-\tau))}{g_{1}(y(x-\tau))}\right) d x\right) d s \\
& +\int_{\sigma_{y}}^{1-a} \Phi^{-1}\left(\int_{\sigma_{y}}^{s} q(x) g_{1}(y(x-\tau))\left(1+\frac{h_{1}(y(x-\tau))}{g_{1}(y(x-\tau))}\right) d x\right) d s \\
& \geq \Phi^{-1}\left(g_{1}\left(R_{2}\right)\right) \Phi^{-1}\left(1+\frac{h_{1}\left(a(a+\tau) R_{2}\right)}{g_{1}\left(a(a+\tau) R_{2}\right)}\right) \\
& \times \min _{\tau+a \leq t \leq 1-a}\left\{\int_{\tau+a}^{t} \Phi^{-1}\left(\int_{s}^{t} q(x) d x\right) d s+\int_{t}^{1-a} \Phi^{-1}\left(\int_{t}^{s} q(x) d x\right) d s\right\} \\
& =2 B_{0} \Phi^{-1}\left(g_{1}\left(R_{2}\right)\right) \Phi^{-1}\left(1+\frac{h_{1}\left(a(a+\tau) R_{2}\right)}{g_{1}\left(a(a+\tau) R_{2}\right)}\right) \\
& >2 R_{2}=2\|y\| .
\end{aligned}
$$

If $1-a \leq \sigma_{y}<1$, then

$$
\begin{aligned}
\|A y\| & =(A y)\left(\sigma_{y}\right)=\int_{0}^{\sigma_{y}} \Phi^{-1}\left(\int_{s}^{\sigma_{y}} q(x) f(x, y(x-\tau)+w(x-\tau)) d x\right) d s \\
& \geq \int_{\tau+a}^{1-a} \Phi^{-1}\left(\int_{s}^{1-a} q(x) f(x, y(x-\tau)) d x\right) d s
\end{aligned}
$$




$$
\begin{aligned}
& \geq \int_{\tau+a}^{1-a} \Phi^{-1}\left(\int_{s}^{1-a} q(x) g_{1}(y(x-\tau))\left(1+\frac{h_{1}(y(x-\tau))}{g_{1}(y(x-\tau))}\right) d x\right) d s \\
& \geq \Phi^{-1}\left(g_{1}\left(R_{2}\right)\right) \Phi^{-1}\left(1+\frac{h_{1}\left(a(a+\tau) R_{2}\right)}{g_{1}\left(a(a+\tau) R_{2}\right)}\right) \int_{\tau+a}^{1-a} \Phi^{-1}\left(\int_{s}^{1-a} q(x) d x\right) d s \\
& \geq B_{0} \Phi^{-1}\left(g_{1}\left(R_{2}\right)\right) \Phi^{-1}\left(1+\frac{h_{1}\left(a(a+\tau) R_{2}\right)}{g_{1}\left(a(a+\tau) R_{2}\right)}\right) \\
& >R_{2}=\|y\| .
\end{aligned}
$$

Thus $\|A y\|>\|y\|$, so (3.35) holds.

Now Lemma 1.2 implies $A$ has a fixed point $y_{2} \in K \cap\left(\bar{\Omega}_{2} \backslash \Omega_{1}\right)$, i.e. $r \leq\left\|y_{2}\right\|=\left\|y_{2}\right\|_{[0,1]} \leq R_{2}$ and $y_{2}(t) \geq t(1-t) r$ for $t \in[0,1]$. It follows from (3.34) and (3.35) that $\left\|y_{2}\right\| \neq r,\left\|y_{2}\right\| \neq R_{2}$, so $r<\left\|y_{2}\right\|<R_{2}$.

Similarly, if we put

$$
\Omega_{1}=\left\{u \in E:\|u\|<R_{1}\right\}, \quad \Omega_{2}=\{u \in E:\|u\|<r\}
$$

we can show that there exists a solution $y_{1}$ to $(3.30)$ with $y_{1}(t)>0$ for $t \in(0,1)$ and $R_{1}<\left\|y_{1}\right\|<r$.

This completes the proof of Theorem 3.2.

The following result can be extracted from the proof of Theorem 3.2.

Theorem 3.3. Suppose (3.1)-(3.7), (3.9) and (3.28) hold. In addition suppose the following condition is satisfied:

(3.36) there exists $0<R_{1}<r$ with

$$
\frac{R_{1}}{\Phi^{-1}\left(g_{1}\left(R_{1}\right)\right) \Phi^{-1}\left(1+\frac{h_{1}\left(a(a+\tau) R_{1}\right)}{g_{1}\left(a(a+\tau) R_{1}\right)}\right)}<B_{0}
$$

where

$$
B_{0}=\frac{1}{2} \min _{\tau+a \leq t \leq 1-a}\left\{\int_{\tau+a}^{t} \Phi^{-1}\left(\int_{s}^{t} q(x) d x\right) d s+\int_{t}^{1-a} \Phi^{-1}\left(\int_{t}^{s} q(x) d x\right) d s\right\} .
$$

Then (1.1) has a nonnegative solution $y \in C[-\tau, 1] \cap C^{1}[0,1]$ with $y(t)>0$ for $t \in(0,1)$ and $R_{1}<\|y-w\|<r$, where $w$ is as in Theorem 3.1.

REMARK 3.3. If in (3.36) we have $R_{1}>r$ then (1.1) has a nonnegative solution $y \in C[-\tau, 1] \cap C^{1}[0,1]$ with $y(t)>0$ for $t \in(0,1)$ and $r<\|y-w\|$ $<R_{1}$.

It is easy to use Theorem 3.3 and Remark 3.3 to obtain theorems which guarantee the existence of more than two solutions to (1.1). We state one such result. 
Theorem 3.4. Suppose (3.1)-(3.7), and (3.28) hold. Assume that there exists $m \in\{1,2, \ldots\}$ and constants $R_{i}, r_{i}(i=1, \ldots, m)$ with $r_{1}>b_{0}$ and

$$
0<R_{1}<r_{1}<R_{2}<r_{2}<\ldots<R_{m}<r_{m} .
$$

In addition suppose that for each $i=1, \ldots, m$,

$$
\frac{r_{i}}{\Phi^{-1}\left(g\left(r_{i}\right)+h\left(r_{i}\right)\right)}>a_{0}
$$

and

$$
\frac{R_{i}}{\Phi^{-1}\left(g_{1}\left(R_{i}\right)\right) \Phi^{-1}\left(1+\frac{h_{1}\left(a(a+\tau) R_{i}\right)}{g_{1}\left(a(a+\tau) R_{i}\right)}\right)}<B_{0},
$$

where $B_{0}$ is as in Theorem 3.3. Then (1.1) has nonnegative solutions $y_{1}, \ldots$ $\ldots, y_{2 m-1} \in C[-\tau, 1] \cap C^{1}[0,1]$ with $y_{i}(t)>0(i=1, \ldots, 2 m-1)$ for $t \in(0,1)$ and

$$
R_{1}<\left\|y_{1}-w\right\|<r_{1}<\ldots<R_{m}<\left\|y_{2 m-1}-w\right\|<r_{m}
$$

where $a_{0}, b_{0}$ and $w$ are as in Theorem 3.1.

EXAmple 3.2. Consider the boundary value problem $(p=2)$

$$
\left\{\begin{array}{l}
y^{\prime \prime}(t)+\sigma\left(y^{-\alpha}(t-\tau)+y^{\beta}(t-\tau)\right)=0, \quad t \in(0,1) \backslash\{\tau\} \\
y(t)=-t, \quad-\tau \leq t \leq 0, \\
y(1)=0, \quad 0<\alpha<1<\beta, 0<\tau<1
\end{array}\right.
$$

where $\sigma>0$ is such that

$$
\begin{aligned}
\sigma<\min \left(\left[\int _ { 0 } ^ { \tau } x \left[(\tau-x)^{-\alpha}+\right.\right.\right. & \left.\left.(\tau-x)^{\beta}\right] d x\right]^{-1}, \\
& {\left.\left[2 \int_{\tau}^{1}(1-x)(x-\tau)^{-\alpha}(1+\tau-x)^{-\alpha} d x\right]^{-1}\right) . }
\end{aligned}
$$

Then (3.39) has two solutions $y_{1}, y_{2}$ with $y_{i}(t)>0$ for $t \in(0,1), i=1,2$.

To see this, we will apply Theorem 3.2 with

$$
\begin{gathered}
g(y)=g_{1}(y)=y^{-\alpha}, \quad h(y)=h_{1}(y)=y^{\beta}, \quad q(t)=\sigma, \\
\xi(t)=-t, \quad K_{0}=1, \quad a=\frac{1-\tau}{4}
\end{gathered}
$$

$\left(0<R_{1}<1<R_{2}\right.$ will be chosen below). Clearly (3.1)-(3.7) and (3.28) hold. Next notice that (see Example 3.1)

$$
\begin{aligned}
& b_{0}=\sigma \int_{0}^{\tau} x\left[(\tau-x)^{-\alpha}+(\tau-x)^{\beta}\right] d x, \\
& a_{0}=\sigma \int_{\tau}^{1}(1-x)(x-\tau)^{-\alpha}(1+\tau-x)^{-\alpha} d x,
\end{aligned}
$$


so (3.9) is true with $r=1$ since

$$
b_{0}<r=1, \quad a_{0}<\frac{1}{2}=\frac{r}{g(r)+h(r)} .
$$

Finally, notice that (3.29) is satisfied for $R_{1}$ small and $R_{2}$ large since

$$
\frac{R_{i}}{g_{1}\left(R_{i}\right)\left\{1+\frac{h_{1}\left(a(a+\tau) R_{i}\right)}{g_{1}\left(a(a+\tau) R_{i}\right)}\right\}}=\frac{R_{i}^{1+\alpha}}{1+a^{\alpha+\beta}(a+\tau)^{\alpha+\beta} R_{i}^{\alpha+\beta}} \rightarrow 0
$$

as $R_{1} \rightarrow 0$ and $R_{2} \rightarrow \infty$, since $\beta>1$. Thus all the conditions of Theorem 3.2 are satisfied, so existence is guaranteed.

\section{References}

[1] R. P. Agarwal and D. O'Regan, Singular boundary value problems for superlinear second order ordinary and delay differential equations, J. Differential Equations 130 (1996), 335-355.

[2] - - - Existence theorems for single and multiple solutions to singular positone boundary value problems, ibid. 175 (2001), 393-414.

[3] - - - Twin solutions to singular Dirichlet problems, J. Math. Anal. Appl. 240 (1999), 433-445.

[4] — - Twin solutions to singular boundary value problems, Proc. Amer. Math. Soc. 128 (2000), 2085-2094.

[5] -, 一, Multiplicity results for singular conjugate, focal, and $(n, p)$ problems, J. Differential Equations 170 (2001), 142-156.

[6] K. Deimling, Nonlinear Functional Analysis, Springer, 1985.

[7] L. H. Erbe and Q. K. Kong, Boundary value problems for singular second order functional differential equations, J. Comput. Appl. Math. 53 (1994), 377-388.

[8] D. Q. Jiang, Multiple positive solutions for boundary value problems of second-order delay differential equations, Appl. Math. Lett. 15 (2002), 575-583.

[9] - Multiple positive solutions to singular boundary value problems for superlinear higher-order ODEs, Comput. Math. Appl. 40 (2000), 249-259.

[10] D. Q. Jiang and H. Z. Liu, On the existence of nonnegative radial solutions for p-Laplacian elliptic systems, Ann. Polon. Math. 71 (1999), 19-29.

[11] D. Q. Jiang and J. Y. Wang, On boundary value problems for singular second-order functional differential equations, J. Comput. Appl. Math. 116 (2000), 231-241.

[12] D. Q. Jiang and P. X. Weng, Multiple positive solutions for boundary value problem of second-order FDE of mixed type, Dynam. Contin. Discrete Impuls. Systems 7 (2000), 561-576.

[13] L. Kong and J. Y. Wang, Multiple positive solutions for the one-dimensional pLaplacian, Nonlinear Anal. 42 (2000), 1327-1333.

[14] D. O'Regan, Some general existence principles and results for $\left(\phi\left(y^{\prime}\right)\right)^{\prime}=q f\left(t, y, y^{\prime}\right)$, $0<t<1$, SIAM J. Math. Anal. 24 (1993), 648-668.

[15] - Existence Theory for Nonlinear Ordinary Differential Equations, Kluwer, Dordrecht, 1997.

[16] J. Y. Wang, The existence of positive solutions for the one-dimensional p-Laplacian, Proc. Amer. Math. Soc. 125 (1997), 2275-2283. 
[17] J. Y. Wang and W. J. Gao, A singular boundary value problem for the one-dimensional p-Laplacian, J. Math. Anal. Appl. 201 (1996), 851-866.

[18] P. X. Weng and D. Q. Jiang, Existence of positive solutions for boundary value problem of second-order FDE, Comput. Math. Appl. 37 (1999), 1-9.

Department of Mathematics

Northeast Normal University

Changchun 130024, P.R. China

E-mail: sxxi@nenu.edu.cn

Department of Mathematics

National University of Ireland

Galway, Ireland

E-mail: donal.oregan@nuigalway.ie
Department of Mathematical Sciences Florida Institute of Technology Melbourne, Florida 32901-6975, U.S.A. E-mail: agarwal@fit.edu

Reçu par la Rédaction le 23.9.2002

Révisé le 13.1.2003 